\title{
A Novel Structure for Design and simulation of a MEMS Resonator for Biomedical Applications
}

\author{
F Setoudeh* \\ Department of Electrical Engineering, Arak University of Technology, Arak, Iran \\ *Corresponding author: F Setoudeh, Department of Electrical Engineering, Iran, Email: F.Setoudeh@arakut.ac.ir
}

\begin{tabular}{|c|c|}
\hline ARTICLE INFO & ABSTRACT \\
\hline Received: 幽 May 27, 2019 & $\begin{array}{l}\text { Micro resonators have found a broad usage in mass sensing applications especially in } \\
\text { biomedical field. }\end{array}$ \\
\hline $\begin{array}{l}\text { Citation: F Setoudeh. A Novel Structure } \\
\text { for Design and simulation of a MEMS } \\
\text { Resonator for Biomedical Applications. } \\
\text { Biomed J Sci \& Tech Res 20(2)-2019. } \\
\text { BJSTR. MS.ID.003428. }\end{array}$ & $\begin{array}{l}\text { This paper aimed to evaluate a micro resonator. In the design of micro resonators, } \\
\text { some factors as micro resonator structure should be considered, actuator and applied cas- } \\
\text { es as it has the highest effect on quality coefficient and consumption power and work fre- } \\
\text { quency. This study at first designs resonator and investigates various structures. At first, a } \\
\text { mechanical analysis is performed by Comsol } 3.5 \text { software on micro resonator to optimize it } \\
\text { in terms of dimensions. The voltage of bias on micro resonator is } 1.1 \mathrm{~V} \text { and switching time } \\
\text { is } 120 \text { microseconds. }\end{array}$ \\
\hline
\end{tabular}

Keywords: Micro resonator; Modeling; Micro Electro; Mechanic Systems

\section{Introduction}

Biomedical devices can be used in applications ranging from neural [1] up to endoscopic capsule systems with video transmission [2]. The achievement of such systems can be said in part with the achievement of micro technologies, which allowed the miniaturization of sensors and actuators followed by their integration with readout and communication electronics. Wireless implantable Microsystems vocalize a breakthrough in the way the interior pathologies can be demeaned. For this purpose, the radiofrequency (RF) chips can play an important role. RF Filter (RF) is of great importance in telecommunication systems (e.g. filters in radars and test systems and measurement). The reason of importance of RF filters at micrometer frequency scale and millimeter waves is due to the limitation of frequency spectrum and frequency spectrum division for various applications [3]. Based on increasing need to more frequency spectrum in radio frequencies and less application to each frequency spectrum, microwaves are more used and applied frequency range in various applications has reached some $10 \mathrm{GHz}$. Using compressed elements in filters design in high frequency is with some problems. One of the problems is that compressed elements as capacitor and inductor have high loss at high frequency and lose their efficiency. The mentioned problems regarding previous circuits caused that engineers apply new methods and make much effort for optimization of circuit in terms of size (or volume ) of circuit, power and feed voltage, noise and economical aspects. In recent years, with the emergence of integrated circuits and development of their technology, great steps are taken for filters development and new filters are made based on Micro electromechanical systems (MEMS) as coping with the mentioned limitations. Micro electro mechanic systems are combination of mechanic and electronic systems in micrometer dimensions (micro electromechanical systems at micrometer dimensions are called MEMS) and even nanometer (micro electromechanical systems at nanometer dimensions, NEMS is called) [4,5]. MEMS devices are widely used in biomedical applications $[6,7]$. Some of these uses include:

a) MEMS in precision surgery-ophthalmology

b) MEMS in biomolecular recognition

c) MEMS in autonomous therapy management systemsmicro-pumps for drug delivery [7].

The main part of MEMS filters is its resonators. Resonators with internal loss and low launch and high-quality coefficient and high frequency stability play important role in design of MEMS filters. Thus, design of simple and cheap resonators is necessary to make MEMS filters [8,9]. This paper evaluates different resonators and advantages and disadvantages of each of them are defined and 
math equations for each of them are extracted. Then, we design micro resonators and the simple resonator is proposed, with low internal loss, high quality coefficient and low launching power and finally the proposed filter is proposed. In the study of Gudarzi et al., electrostatic micro resonators are introduced and at best state for $7 \mathrm{~V}$ voltage, 2.5 micrometer displacement is created [10]. In the study done by Mingxin et al., an electrostatic micro resonator is introduced in which actuation voltage is reduced [11]. To fulfill low actuation voltage, beams are spiral. By changing the width and thickness of beams, actuation voltage is reduced and voltage $1.5 \mathrm{~V}$ is obtained for aerial distance $2.5 \mu \mathrm{m}$ [11].

In [12], electrostatic micro resonators are introduced as at best state presenting displacement $2.5 \mu \mathrm{m}$ for voltage $3.5 \mathrm{~V}$.

In [13], electrostatic micro resonators are introduced in which beams are connected as butterfly and at best state, for voltage $11 \mathrm{~V}$, displacement 3micrometer is presented [13]. In [14], two types of electrostatic micro resonators are introduced and at best stage for voltage $1.5 \mathrm{~V}$, presents displacement 0.5 micrometer [14]. In the study of Ya et al., (2013), electrostatic micro resonator is introduced applying four beams for suspending mobile electrode [15]. t This Resonator at best state for voltage $3 \mathrm{~V}$ presents displacement 2.2 $\mu \mathrm{m}$ [15]. As shown, actuation mechanism for micro resonator is selected based on design regulations. Generally, piezoelectric actuators have low consumption power and high speed, but they have difficult construction process and they need high actuation voltage [16]. Magnetic actuators have difficult construction process and are minimized hardly, and they have costly construction process [17]. Electro thermal actuators can be generated easily by standard Micro fabrication techniques in Monolithic designs. Electro thermal actuators work at high temperature and have high thermal loss [18]. Electrostatic actuators have easy construction process and are economical. Also, they have low consumption power, high speed, high controlling compared to other actuators [19]. But electrostatic actuators have high actuation voltage. Actuation voltage is not a basic limitation for electrostatic actuator, and we can introduce optimize design reducing actuation voltage [19]. As it was said, electrostatic actuators are the best choice to design resonator in the present study.

In [20], the Melnikov method is used to pull-in instability analysis of an electrostatic MEMS resonator [20]. In [21], the efficacy of surface processing error on the thickness is considered to analysis of MEMS resonator [21].

Various processes are used in construction of MEMS as Wafer cleaning, Lithography, Diffusion, Deposition, Etching and Ion Implanting [22]. In the design of a MEMS, materials selection is of great importance as its structure [23]. As first, materials determined construction method and has direct effect on practicality of plan. Second, materials affect electric and mechanic features and have great effect on parameters as consumption power, loss and return $[23,24]$. The starting point of this paper is fulfilling a filter eliminating unwanted signals. Thus, this filter is made of some resonators as fluctuating via an actuation mechanism in specific mechanism. Thus, some specific needs of design should be considered to select the best structure:

a) Having low consumption power

b) Suitable limit of resonator displacement

c) High response speed

d) Controlling

e) Performance at relatively low temperature to avoid thermal loss

f) Isolating actuation mechanism (actuator is as insulated as thermal and electric)

g) Low noise

h) Plan Disposability

i) Easy construction: Integration with electronic circuits can reduce construction costs. The possibility of plan depends upon construction process.

\section{Data Analysis Structural Investigation of Resonator}

Electrostatic actuators are the best choice for micro resonators. Figure 1 shows schematic of simple electrostatic actuation model. In this model, it is assumed that electrodes are complete. Also, it is assumed that capacitor structure is located in vacuum to guarantee external mechanic loading as zero for upper electrode. Figure 2 shows the 3D model of resonator [25]. By increased but beams (for construction problems) are not reduced more than a size. By creating some holes on movable plate, resonator return is increased. By reduction of aerial distance and thickness of movable plates, we can increase actuator return but due to construction problems, it is not reduced more than a size [26]. The best choice to increase resonator return is changes in structure and dimensions of beams and creating some holes on movable plate with highest effect on return. Thus, Figure 3 proposed the structure of micro resonators with holes with long beams [3]. Displacement distribution on micro resonator at bias voltage $12 \mathrm{~V}$ is as Figure 4 and it has the highest displacement at center as 2.41 micrometer. Displacement changes chart in movable plat based on voltage changes is shown in Figure 5 . Based on chart, the highest displacement is made in voltage $12 \mathrm{~V}$. Also, the chart of displacement changes based on time changes is shown in Figure 6 and based on switching speed chart, this resonator is about 60 microseconds. Based on the results, we can say the designed structure has high return compared to previous states. In the next section, we optimize this plan [26]. 


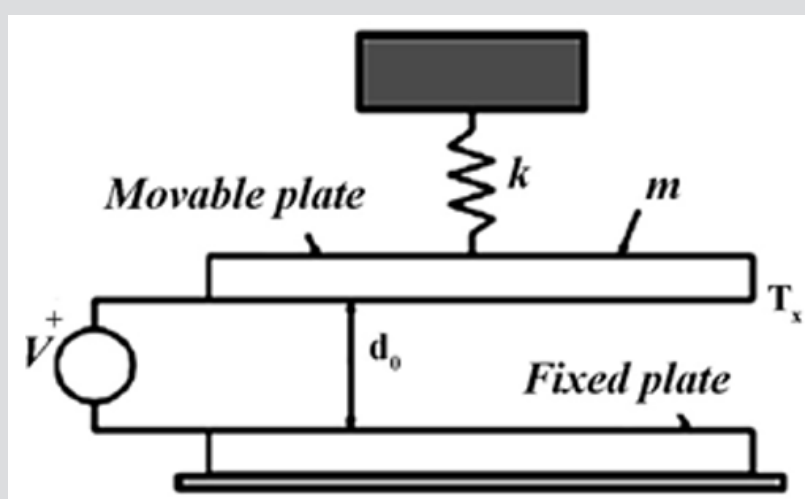

Figure 1: Simple electrostatic actuation model.

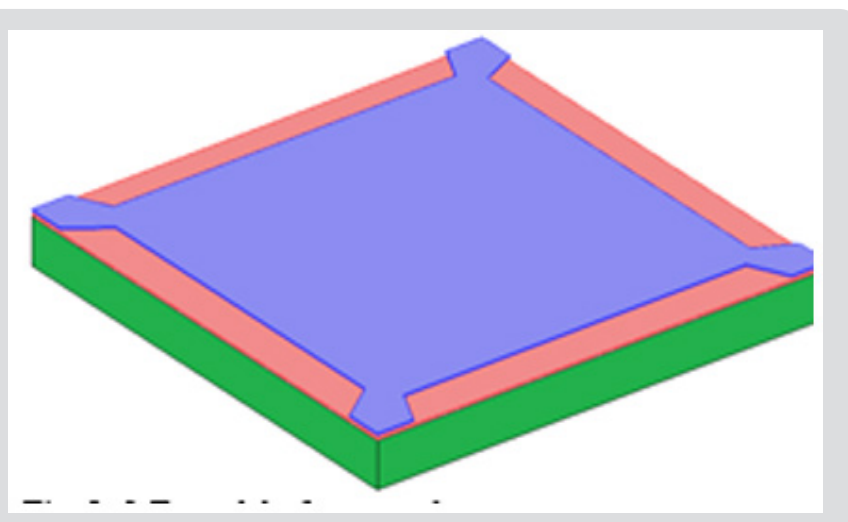

Figure 2: 3-D model of proposed structure resonator.

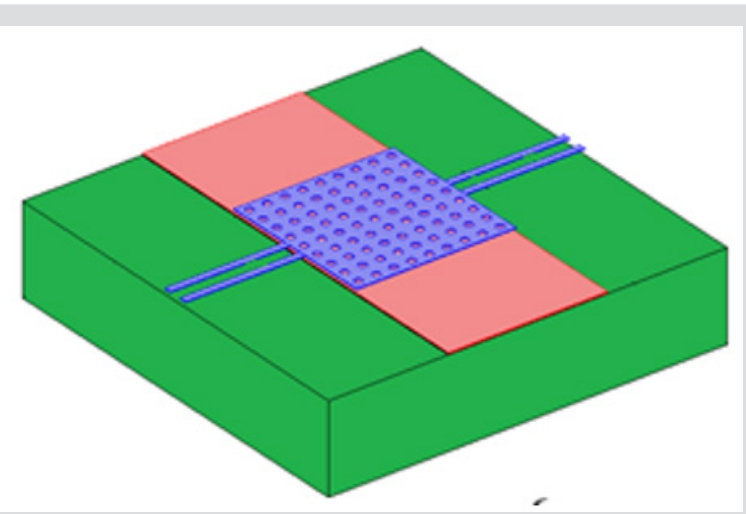

Figure 3: Schematic of micro resonators with holes with long beams.

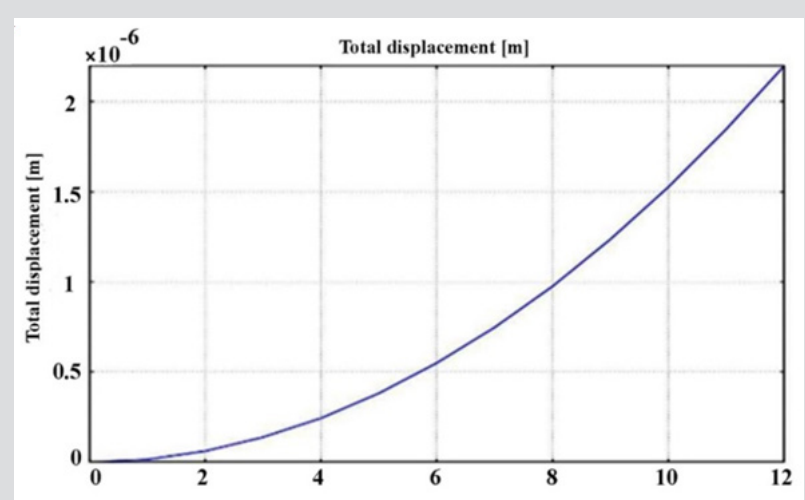

Figure 4: The displacement chart in terms of voltage changes in micro resonators with holes and long beams.

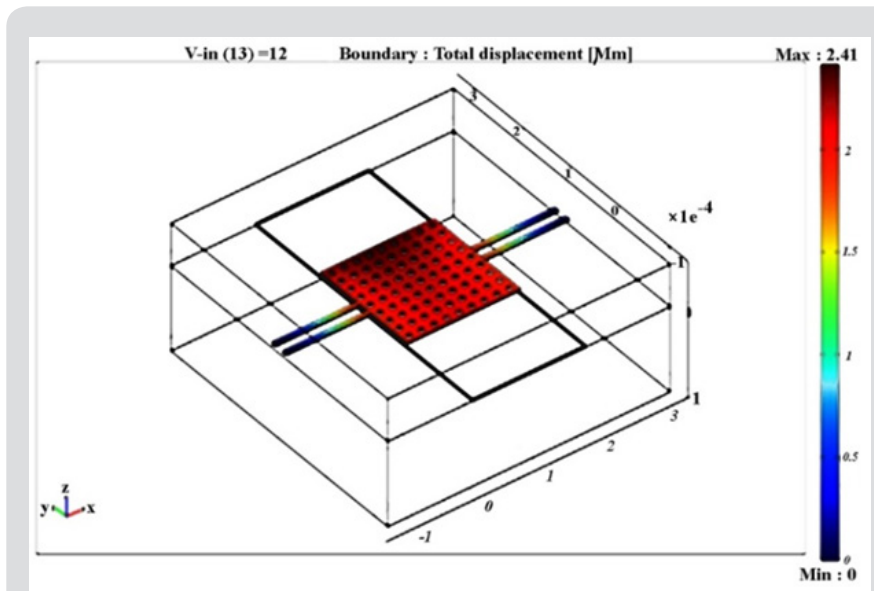

Figure 5: Displacement distribution at bias voltage 12V.

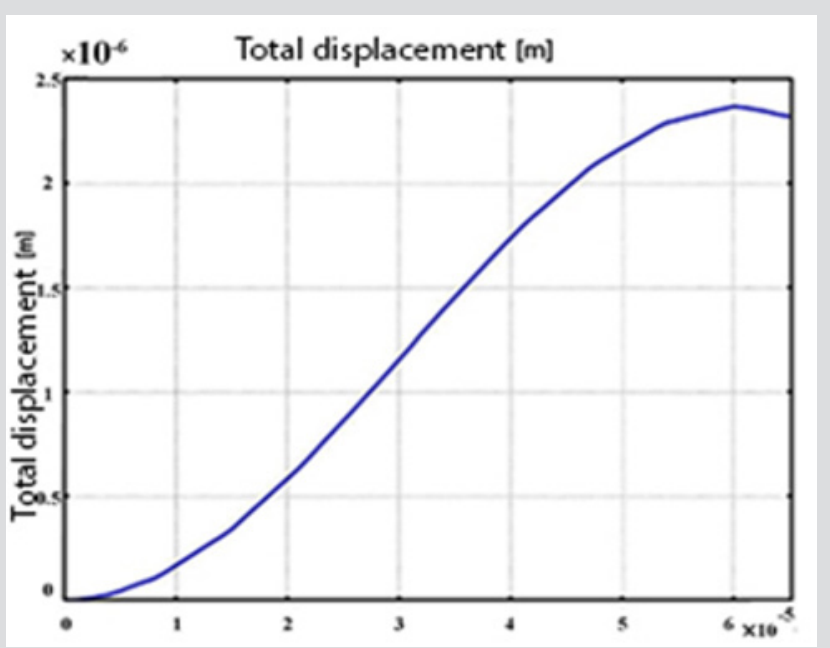

Figure 6: The time analysis chart of micro resonator with holes with long beams.

\section{Proposed Structure for Modified Micro Resonator}

This section introduces optimized micro resonator and important factors of this actuator as voltage, displacement and switching speed are discussed. The Figure of displacement changes in movable plat based on voltage changes is shown in Figure 7. Based on the chart, the highest displacement is in voltage $1.1 \mathrm{~V}$. The schematic of optimized micro resonators is shown in Figure 8. As shown in Figure 7, micro resonator structure is composed of two fixed and movable plates made of aluminum. Thickness of movable and fixed plate is 1, 0.5 micrometer and aerial distance between two plates is 2.5 micrometer. The bed and sub-layer are made of silicon and silicon dioxide. The beams of this micro resonator are spiral, and the return is increased. The Figure of displacement changes in movable plat based on voltage changes is shown in Figure 8. Based on the chart, the highest displacement is in voltage 1.1V. Displacement distribution on micro resonators in bias voltage $1.1 \mathrm{~V}$ is shown in Figure 9 and highest. Also, the chart of displacement changes based on time variant is shown in Figure 10 and based on switching speed chart of this resonator is about 160 microseconds. Temperature distribution is shown in structure of Figure 11. 


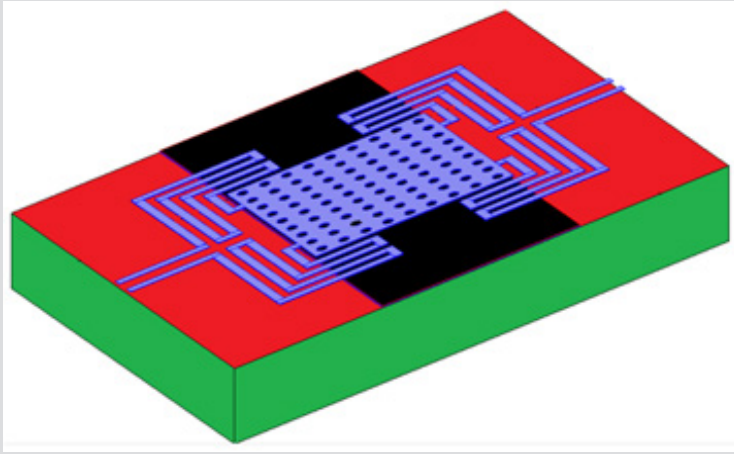

Figure 7: Schematic of optimized micro resonator.

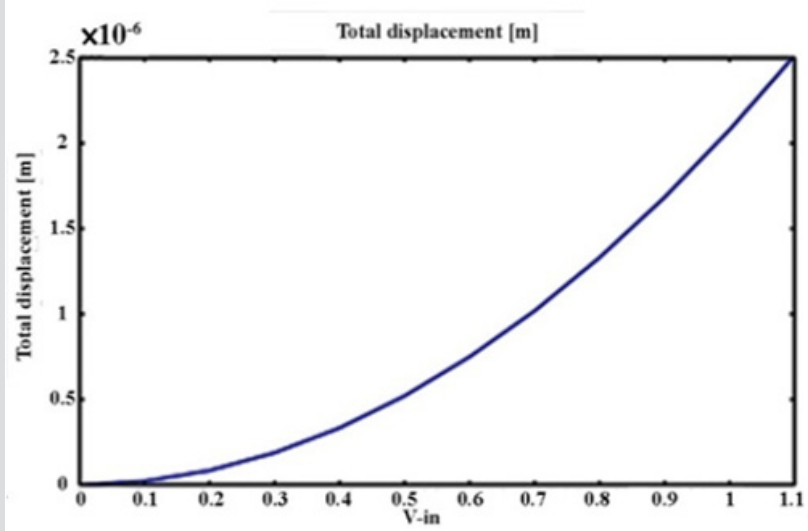

Figure 8: The displacement chart in terms of voltage changes in optimized micro resonators.

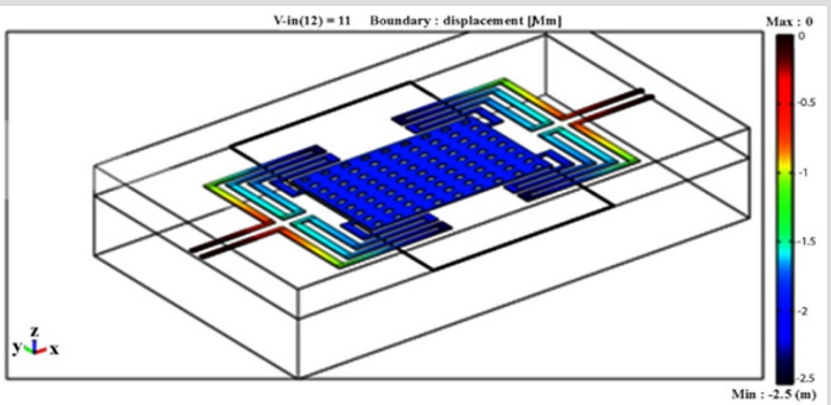

Figure 9: Displacement distribution in bias voltage 1.1V.

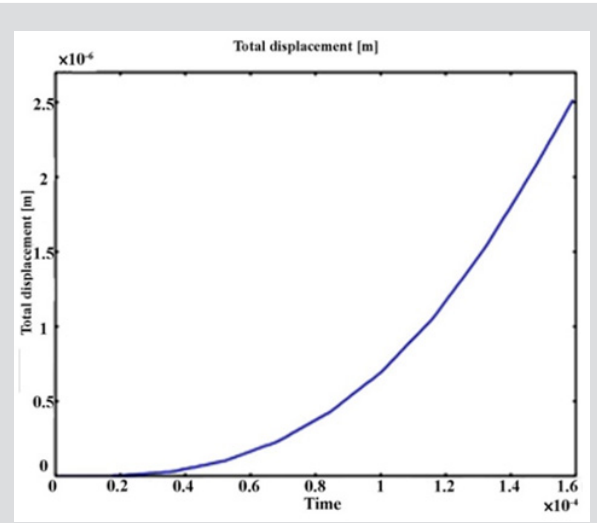

Figure 10: The chart of time analysis of optimized micro resonator.

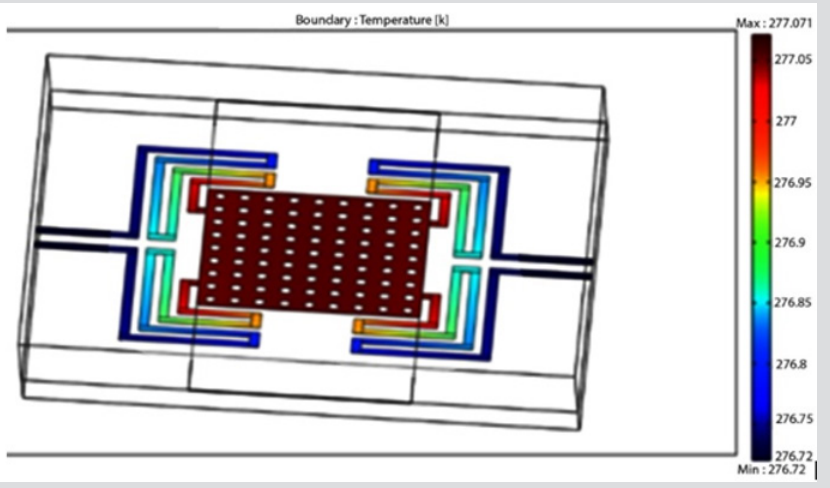

Figure 11: Temperature distribution in Kelvin degree.

\section{Conclusion}

To design micro resonator, the followings are made:

1. The basic part of a micro resonator is its actuator and at first, various actuators were investigated and after that, it was found that electrostatic actuators are the best choice to design micro resonator as they have easy construction process.

2. For simulation of micro resonator with electrostatic actuator, some samples are proposed and simulated and various parameters as dimensions, structure and constituent materials were changed and the effect of these changes on micro resonator parameters was investigated.

3. Simulation results of optimized model of micro resonator are shown in the Table 1.

Table 1: The simulation results of optimized micro resonator.

\begin{tabular}{|c|c|c|c|}
\hline $\begin{array}{c}\text { Switching } \\
\text { speed }\end{array}$ & $\begin{array}{c}\text { Generated } \\
\text { displacement }\end{array}$ & $\begin{array}{c}\text { Applied } \\
\text { voltage }\end{array}$ & $\begin{array}{c}\text { Micro resonator } \\
\text { dimensions }\end{array}$ \\
\hline 160 (us) & $2.5(\mathrm{um})$ & $1.1 \mathrm{~V}$ & $120^{*} 500^{*} 700(\mathrm{um})$ \\
\hline
\end{tabular}

The comparison of the results of designed micro resonator with the previous works is shown in Table 2.

Table 2: Comparison of the results of designed micro resonator with the previous works.

\begin{tabular}{|c|c|c|}
\hline & Displacement (um) & Applied voltage \\
\hline Reference [4] & 2.5 & $7 \mathrm{~V}$ \\
\hline Reference [11] & 2.5 & $1.5 \mathrm{~V}$ \\
\hline Reference [12] & 2.5 & $3.5 \mathrm{~V}$ \\
\hline Reference [13] & 3 & $11 \mathrm{~V}$ \\
\hline Reference [14] & 0.5 & $1.5 \mathrm{~V}$ \\
\hline Reference [15] & 2.2 & $3 \mathrm{~V}$ \\
\hline Presented work & 2.5 & $1.1 \mathrm{~V}$ \\
\hline
\end{tabular}

\section{References}

1. K Lau, S Lee, P Chan (1999) A low-power synapse/neuron cell for artificial neural networks. Microelectronics journal 30(12): 1261-1264.

2. B Chi, J Yao, S Han, X Xie, G Li, Z Wang (2007) Low power high data rate wireless endoscopy transceiver. Microelectronics Journal 38(1011):1070-1081. 
3. FD Bannon,JRClark, CCNguyen(2000)High-QHF microelectromechanical filters. IEEE Journal of solid-state circuits 35(4):512-526.

4. S Diamantis, M Ahmadi, G Jullien, W Miller (2000) A programmable MEMS bandpass filter in Proceedings of the 43rd IEEE Midwest Symposium on Circuits and Systems (Cat. No. CH37144) 1: 522-525.

5. MK Chaubey, A Bhadauria (2018) RF MEMS Based Tunable Bandpass Filter For X-Band Applications," in IOP Conference Series: Materials Science and Engineering 331(1): 012030.

6. DL Polla (2001) MEMS technology for biomedical applications, in $20016^{\text {th }}$ International Conference on Solid-State and Integrated Circuit Technology. Proceedings (Cat. No. 01EX443) 1: 19-22.

7. Nisar, N Afzulpurkar, B Mahaisavariya, A Tuantranont (2008) MEMSbased micropumps in drug delivery and biomedical applications. Sensors and Actuators B: Chemical 130(2): 917-942.

8. AA Kumar, T Shanmuganantham, MS Reddy (2016) Performance analysis of MEMS resonator for filter application. in 2016 International Conference on Control, Instrumentation, Communication and Computational Technologies (ICCICCT) IEEE: 242-245.

9. S Ilyas, N Jaber, MI Younis (2018) A MEMS coupled resonator for frequency filtering in air. Mechatronics 56: 261-267.

10. G Saeed, G Ashkan, A Amin (2013) Designing a RFMEMS switch with a spiral bend and a gold membrane to reduce the excitation voltage. Can J Electr Electron Eng 4(1)

11. M Song, J Yin, X He, Y Wang (2008) Design and analysis of a nove low actuation voltage capacitive RF MEMS switches in 2008 3rd IEEE International Conference on Nano/Micro Engineered and Molecular Systems 2008: 235-238.

12. ML Ya, N Soin, AN Nordin (2014) Novel low-voltage RF-MEMS switch: Design and simulation," in 2014 IEEE International Conference on Semiconductor Electronics (ICSE2014) pp: 142-145.

13. BL Reddy, T Shanmuganantham (2014) Design of novel capacitive RF MEMS shunt switch with aluminum nitride (AIN) dielectric. Procedia materials science 6: 692-700.

14. R Kaur, C Tripathi, D Kumar (2014) Low voltage RF MEMS capacitive shunt switches. Wireless personal communications 78(2): 1391-1401.

\section{ISSN: 2574-1241}

DOI: 10.26717/BJSTR.2019.20.003428

F Setoudeh. Biomed J Sci \& Tech Res

(c) (P) This work is licensed under Creative Commons Attribution 4.0 License

Submission Link: https://biomedres.us/submit-manuscript.php
15. ML Ya, AN Nordin, N Soin (2013) Design and analysis of a low-voltage electrostatic actuated RF CMOS-MEMS switch. in RSM 2013 IEEE Regional Symposium on Micro and Nanoelectronics 2013: 41-44.

16. J Toledo, V Ruiz Díez, M Bertke, H Suryo Wasisto, E Peiner, et al. (2019) Piezoelectric MEMS Resonators for Cigarette Particle Detection. Micromachines 10(2):145.

17. MU Mian, JO Dennis, MH Khir, MG Ahmed AA Rabih, et al. (2016) Optical and capacitive characterization of MEMS magnetic resonator. IEICE Electronics Express 13(18): 20160773-20160773.

18. W Suocheng, H Yongping, L Shuangjie (2015) The design and analysis of a MEMS electrothermal actuator. Journal of Semiconductors 36(4): 044012 .

19.S Gorthi (2006) Characterization of MEMS Electrostatic Actuators Beyond Pull-In.

20. H Shang (2017) Pull-in instability of a typical electrostatic MEMS resonator and its control by delayed feedback. Nonlinear Dynamics 90(1): 171-183.

21.J Feng, C Liu, W Zhang, S Hao (2018) Static and dynamic mechanical behaviors of electrostatic MEMS resonator with surface processing error. Micromachines 9(1): 34 .

22. AA Caballero, KK Yen (2003) The Use of Micro-Electro-Mechanical Systems (MEMS) in the Construction Industry. Florida International University pp. 161-165.

23. R Pratap, A Arunkumar (2007) Material selection for MEMS devices.

24. I Gil, F Martın, X Rottenberg, W De Raedt (2007) Tunable stop-band filter at Q-band based on RF-MEMS metamaterials," Electronics Letters 43(21): 1153-1154.

25. J. Brotz (2004) Damping in CMOS-MEMS resonators. Masters Project Report in ECE. Carnegie Mellon University.

26. J Giner, A Uranga, F Torres, E Marigo, N Barniol (2010) Fully CMOS integrated bandpass filter based on mechanical coupling of two RF MEMS resonators. Electronics letters 46(9): 640-641.

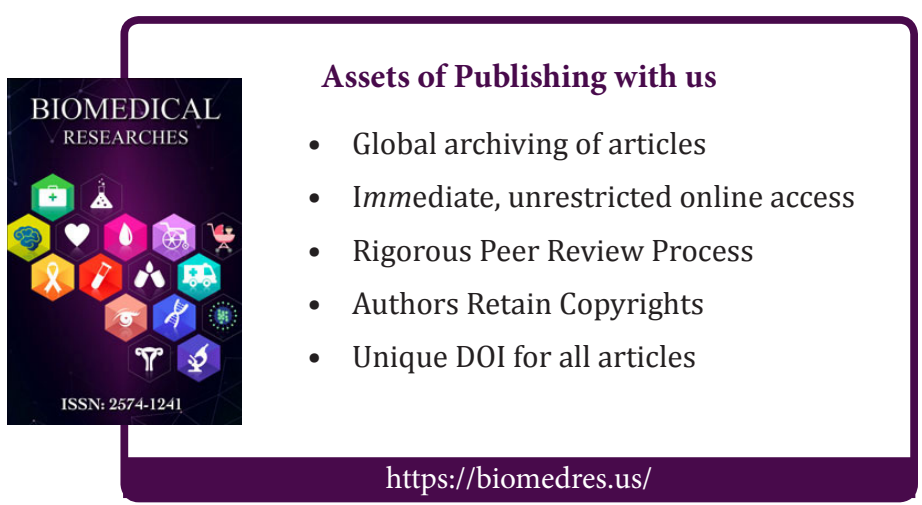

